\title{
BMJ Open Association between chronic obstructive pulmonary disease and increased risk of benign prostatic hyperplasia: a retrospective nationwide cohort study
}

\author{
Yi-Hao Peng, ${ }^{1,2,3}$ Chien-Wen Huang, ${ }^{4,5,6}$ Wei-Chih Liao, ${ }^{7,8}$ Hsuan-Ju Chen,,${ }^{8,9}$ \\ Ming-Chien Yin, ${ }^{1,10}$ Yu-Ming Huang, ${ }^{2}$ Trong-Neng Wu, ${ }^{11}$ Wen-Chao Ho ${ }^{1}$
}

To cite: Peng Y-H, Huang C-W, Liao W-C, et al. Association between chronic obstructive pulmonary disease and increased risk of benign prostatic hyperplasia: a retrospective nationwide cohort study. BMJ Open 2017;7:e015581. doi:10.1136/ bmjopen-2016-015581

- Prepublication history for this paper is available online. To view these files, please visit the journal online (http://dx.doi org/10.1136/bmjopen-2016015581).

Received 16 December 2016 Revised 14 April 2017 Accepted 23 May 2017

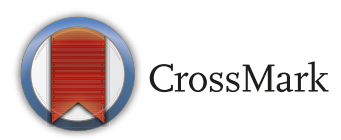

For numbered affiliations see end of article.

Correspondence to

Dr Wen-Chao Ho;

whocmu@gmail.com

\section{ABSTRACT}

Objective Chronic obstructive pulmonary disease (COPD) and benign prostatic hyperplasia (BPH) are common disorders in ageing male populations. Nevertheless, the relationship between the two diseases has rarely been explored. The objective of this study was to examine whether patients with COPD are at an increased risk of $\mathrm{BPH}$.

Design Retrospective nationwide cohort study.

Setting Data retrieved from the Taiwan National Health Insurance Research Database.

Participants Overall, 19959 male patients aged 40 years and over with newly diagnosed COPD between 2000 and 2006 were included as the COPD group, and 19959 sex-matched and age-matched enrollees without COPD were included as the non-COPD group. Both groups were followed-up until the end of 2011.

Outcome measures A Cox proportional hazards regression model was used to compute the risk of $\mathrm{BPH}$ in patients with COPD compared with enrollees without COPD.

Results The overall incidence rate of $\mathrm{BPH}$ was 1.53 times higher in the COPD group than that in the nonCOPD group (44.7 vs 25.7 per 1000 person-years, $95 \% \mathrm{Cl}$ 1.46 to 1.60 ) after adjusting for covariates. An additional stratified analysis revealed that this increased risk of BPH in patients with COPD remained significantly higher than that in enrollees without COPD in all men aged 40 years and over.

Conclusion After adjustment for covariates, male patients with COPD were found to be at a higher risk of BPH. We suggest that clinicians should be cautious about the increased risk of BPH in male patients with COPD.

\section{INTRODUCTION}

Chronic obstructive pulmonary disease (COPD), predominantly prevalent in men, is a chronic inflammatory disorder of the airway and lungs. COPD is one of the most prevalent diseases and the third leading cause of death globally. ${ }^{1}$ Patients with COPD usually present with progressive dyspnoea, shortness of breath and productive cough. Moreover, they frequently experience various comorbid

\section{Strengths and limitations of this study}

This is the first large cohort study to demonstrate that male patients with chronic obstructive pulmonary disease were at a higher risk of benign prostatic hyperplasia.

- The data we used were retrieved from the Taiwan National Health Insurance Research Database, which is highly representative of the general population.

- Detailed patients information, as well as information regarding clinical variables were lacking. Unknown confounding factors might remain, leading to bias in the study results.

conditions, such as cardiovascular disease, metabolic disorder, dementia and skeletal muscle dysfunction. ${ }^{23}$ These comorbidities might have a significant effect on patient outcome. ${ }^{4}$ COPD is currently recognised as a chronic systemic inflammatory state because the inflammation involves the lung and may contribute to various extrapulmonary effects. $^{56}$

Benign prostatic hyperplasia $(\mathrm{BPH})$ is a common medical condition in older male populations. Approximately $14 \%$ of men aged $40-49$ years are estimated to have $\mathrm{BPH}$, and the prevalence increases to $>50 \%$ in men aged 60 years and over. ${ }^{78}$ Patients with $\mathrm{BPH}$ usually present with lower urinary tract symptoms (LUTS), such as urinary urgency and retention, considerably affecting the quality of their lives. In addition to ageing, other reported predisposing factors for BPH are metabolic syndrome, obesity and reduced physical activity. ${ }^{9-11}$

Although COPD and BPH are associated with chronic inflammation of the airway and prostate, respectively, ${ }^{12}{ }^{13}$ and are common disorders in ageing male populations, the relationship between these two conditions has rarely been explored. In addition, previous 
studies have suggested that COPD and BPH might share an underlying pathophysiology. For example, higher levels of interleukin (IL-6) and C reactive protein (CRP) in serum or sputum were observed in both patients with COPD and BPH. ${ }^{14-16}$ Moreover, while patients with COPD appear to be more physically inactive than their counterpart without COPD, ${ }^{17}$ studies have suggested that level of physical activity was negatively associated with BPH risk. This implies that physical inactivity among patients with COPD might also be a contributing factor for BPH development. ${ }^{18}$ In the present study, we investigated whether the risk of BPH increased in patients with COPD by using a retrospective nationwide cohort study design and data retrieved from the Taiwan National Health Insurance Research Database (NHIRD).

\section{METHODS}

\section{Data sources}

The National Health Insurance (NHI) programme is a universal insurance programme established on 1 March 1995, which reformed 13 insurance-related systems to provide comprehensive medical care coverage for up to $99 \%$ of the inhabitants of Taiwan by the end of 2014 . The current study was a population-based cohort study that used data between 1996 and 2011 from the NHIRD, which was established by the National Health Research Institutes (NHRI) with authorisation from the Bureau of National Health Insurance and the Department of Health. In the present study, we used datasets from the Registry for Longitudinal Health Insurance Database 2000 (LHID 2000), which contains medical reimbursement claims data of the NHI programme for 1 million individuals. The NHI reported no differences in the sex and age distributions between the data in the LHID 2000 and beneficiary data in the entire NHI database; thus, the LHID 2000 was considered representative of the general population. The LHID 2000 contains comprehensive information including demographic data, clinical visit dates, diagnostic codes and prescription details. ${ }^{19}$ For security and privacy purposes, patient identity data are encrypted by the NHIRD before releasing them for research purposes.

\section{Study population}

From 1 January 2000 to 31 December 2006, 25036 male patients with COPD were identified from the NHIRD as the potential COPD study population. COPD was diagnosed using the International Classification of Diseases, Ninth Revision, Clinical Modification (ICD-9-CM) codes 491, 492 and 496. To ensure the accuracy of diagnosis, we defined patients as having COPD only when the diagnosis was confirmed at least thrice by outpatient services or inpatient hospitalisation claims. The date of COPD diagnosis was considered as the index date. We excluded patients with missing age or sex information $(n=3)$, those younger than 40 years $(n=4264)$ and those with a history of BPH before the index date $(n=810)$ from the COPD group. For each patient with COPD, one insured individual without COPD was randomly selected from the LHID 2000 to form the non-COPD group, and frequency-matched for sex, 5-year age interval and index year by considering the same inclusion criteria as that for the COPD group.

The demographic factors considered were age (age groups: 40-54, 55-69 and $\geq 70$ years) and annual outpatient department (OPD) visits. Information regarding critical comorbidities of each individual was retrieved from the medical claims based on the ICD-9-CM codes. Comorbidities such as diabetes mellitus (DM, ICD-9-CM code 250), dyslipidemia (ICD-9-CM code 272), hypertension (ICD-9-CM codes 401-405), heart failure (ICD-9-CM code 428) and coronary artery disease (CAD, ICD-9-CM codes 410-414) were obtained before the index date. Patients were considered long-term users of tiotropium or ipratropium if they had been prescribed tiotropium or ipratropium during the follow-up period.

The primary outcome was BPH, which was determined by at least thrice diagnosis confirmations during inpatient hospitalisation or outpatient service, based on the ICD-9-CM diagnosis code 600.0. The follow-up began on the index date and lasted until the first diagnosis date of BPH, withdrawal from NHI or 31 December 2011, whichever occurred first.

\section{Statistical analysis}

Continuous data were summarised using mean and $\mathrm{SD}$, and categorical variables were summarised using frequency and percentage. Continuous variables were compared using a Student's t-test, and the categorical variables were compared using a $\chi^{2}$ test. The incidence rate (per 1000 person-years) of BPH was calculated by dividing the number of $\mathrm{BPH}$ cases by person-time at risk. The cumulative BPH incidence curve was plotted using the Kaplan-Meier method and statistical significance was examined using the log-rank test. Univariate and multivariate Cox proportional hazards regression models were used to analyse the association between BPH-associated risk factors and the incidence of BPH. Age, DM, dyslipidemia, hypertension, heart failure, $\mathrm{CAD}$, tiotropium use, ipratropium use and annual OPD visits were included as covariates in the multivariate Cox regression model. In addition, we performed age-stratified and comorbidity-stratified analyses to estimate the association between COPD and the risk of BPH. HRs and 95\% CIs were calculated to quantify the risk of BPH. The analyses were conducted using SAS V.9.4 (SAS Institute, Cary, North Carolina, USA), and significance was considered as a two-sided $\mathrm{p}<0.05$.

\section{RESULTS}

In this study, 19959 patients with COPD and 19959 enrollees without COPD were enrolled (table 1). The mean ages of the COPD and non-COPD groups were 64.5 years $(\mathrm{SD}=12.2$ years) and 63.8 years $(\mathrm{SD}=12.4$ years $)$, 
Table 1 Comparisons of baseline demographic factors and comorbidities between study enrollees with and without COPD

\begin{tabular}{|c|c|c|c|c|c|}
\hline \multirow[b]{2}{*}{ Variable } & \multicolumn{2}{|c|}{$\begin{array}{l}\text { Non-COPD group } \\
n=19959\end{array}$} & \multicolumn{2}{|c|}{$\begin{array}{l}\text { COPD group } \\
n=19959\end{array}$} & \multirow[t]{2}{*}{ p Value } \\
\hline & $\mathrm{n}$ & $\%$ & $\mathbf{n}$ & $\%$ & \\
\hline Age, years & & & & & $>0.99$ \\
\hline 40-54 & 5141 & 25.8 & 5141 & 25.8 & \\
\hline $55-69$ & 7274 & 36.4 & 7274 & 36.4 & \\
\hline$\geq 70$ & 7544 & 37.8 & 7544 & 37.8 & \\
\hline Mean $(\mathrm{SD})^{\star}$ & 63.8 & $(12.4)$ & 64.5 & (12.2) & $<0.001$ \\
\hline \multicolumn{6}{|l|}{ Comorbidity } \\
\hline DM & 2595 & 13.0 & 3209 & 16.1 & $<0.001$ \\
\hline Dyslipidemia & 3267 & 16.4 & 4621 & 23.2 & $<0.001$ \\
\hline Hypertension & 7672 & 38.4 & 10432 & 52.3 & $<0.001$ \\
\hline Heart failure & 375 & 1.88 & 981 & 4.92 & $<0.001$ \\
\hline CAD & 3141 & 15.7 & 5739 & 28.8 & $<0.001$ \\
\hline \multicolumn{6}{|l|}{ Medication } \\
\hline Use of tiotropium† & 0 & 0.00 & 39 & 0.20 & $<0.001$ \\
\hline Use of ipratropium† & 2 & 0.01 & 135 & 0.68 & $<0.001$ \\
\hline Annual OPD visits & & & & & $<0.001$ \\
\hline$<30$ & 16050 & 80.4 & 10869 & 54.5 & \\
\hline$\geq 30$ & 3909 & 19.6 & 9090 & 45.5 & \\
\hline Mean $(\mathrm{SD})^{\star}$ & 18.9 & (17.1) & 32.8 & (22.4) & $<0.001$ \\
\hline
\end{tabular}

*Student's t-test.

†Fisher's exact test.

CAD, coronary artery disease; COPD, chronic obstructive pulmonary disease; DM, diabetes mellitus; OPD, outpatient department.

respectively. The COPD group had a higher prevalence of comorbidity (including DM, dyslipidemia, hypertension, heart failure and $\mathrm{CAD}$ ) and medications (including tiotropium and ipratropium) than did the non-COPD group. The median annual OPD visits of the COPD and non-COPD groups were $32.8(\mathrm{SD}=22.4)$ and 18.9 $(\mathrm{SD}=17.1)$, respectively.

Cumulative incidence curves of BPH for the COPD and non-COPD groups are presented in figure 1 . The cumulative incidence of $\mathrm{BPH}$ was significantly higher in the COPD group than that in the non-COPD group (log-rank test, $\mathrm{p}<0.001$ ).

During a median follow-up of 6.93 (95\% CI 6.88 to 6.98 ) years, 5690 patients were diagnosed as having BPH in the COPD group, and 3590 patients were diagnosed as having BPH in the non-COPD group, with an incidence rate of 44.7 and 25.7 per 1000 person-years, respectively. Cox proportional hazards regression analysis showed that patients with COPD had a greater risk of BPH than did enrollees without COPD after adjusting for age, comorbidities, medicine use and annual OPD visits (adjusted HR 1.53; $95 \%$ CI 1.46 to 1.60 ; table 2). The risk of $\mathrm{BPH}$ appears to increase with age. Individuals aged 55-69 years and those older than 70 years had adjusted HRs of 2.76 (95\% CI 2.58 to 2.96 ) and 3.84 (95\% CI 3.58 to 4.12), respectively, compared with individuals aged 40-54 years. In addition, dyslipidemia, hypertension,
CAD, ipratropium use and $>30$ annual OPD visits were associated with a higher risk of BPH using multivariate Cox regression analysis.

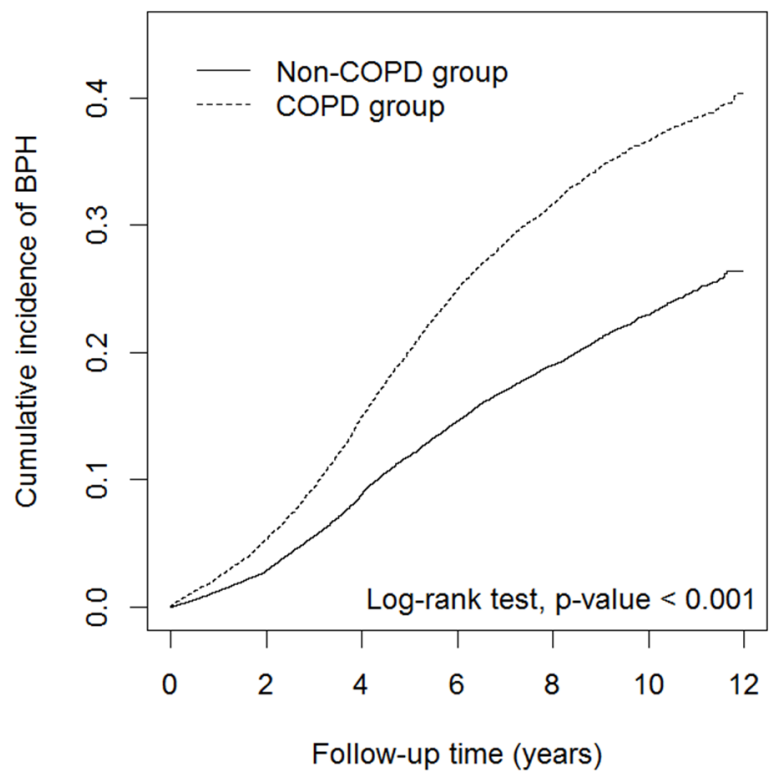

Figure 1 Cumulative incidence curves of benign prostatic hyperplasia for groups with and without COPD. COPD, chronic obstructive pulmonary disease; $\mathrm{BPH}$, benign prostatic hyperplasia. 
Table 2 Cox model measured HRs and 95\% Cl of BPH associated with COPD and covariates

\begin{tabular}{|c|c|c|c|c|c|}
\hline \multirow[b]{2}{*}{ Variable } & \multirow[b]{2}{*}{ Event no. } & \multirow[b]{2}{*}{ Person-years } & \multirow[b]{2}{*}{ IR } & \multicolumn{2}{|l|}{ HR $(95 \% \mathrm{Cl})$} \\
\hline & & & & Univariate & Multivariate $†$ \\
\hline \multicolumn{6}{|l|}{ COPD } \\
\hline No & 3590 & 139853 & 25.7 & 1.00 & 1.00 \\
\hline Yes & 5690 & 127246 & 44.7 & $1.76(1.69-1.83)^{\star \star \star}$ & $1.53(1.46-1.60)^{\star \star \star}$ \\
\hline \multicolumn{6}{|c|}{ Age, years } \\
\hline $40-54$ & 1043 & 84598 & 12.3 & 1.00 & 1.00 \\
\hline $55-69$ & 3898 & 102405 & 38.1 & $3.14(2.93-3.36)^{\star \star \star}$ & $2.76(2.58-2.96)^{* \star \star}$ \\
\hline$\geq 70$ & 4339 & 80097 & 54.2 & $4.63(4.33-4.95)^{\star \star \star}$ & $3.84(3.58-4.12)^{\star \star \star}$ \\
\hline \multicolumn{6}{|c|}{ Comorbidity } \\
\hline \multicolumn{6}{|c|}{ DM } \\
\hline No & 7702 & 233681 & 33.0 & 1.00 & 1.00 \\
\hline Yes & 1578 & 33419 & 47.2 & $1.46(1.38-1.54)^{\star \star \star}$ & $0.99(0.94-1.05)$ \\
\hline \multicolumn{6}{|c|}{ Dyslipidemia } \\
\hline No & 6774 & 216531 & 31.3 & 1.00 & 1.00 \\
\hline Yes & 2506 & 50568 & 49.6 & $1.59(1.52-1.66)^{\star \star \star}$ & $1.24(1.18-1.30)^{\star \star \star}$ \\
\hline \multicolumn{6}{|c|}{ Hypertension } \\
\hline No & 3868 & 158724 & 24.4 & 1.00 & 1.00 \\
\hline Yes & 5412 & 108375 & 49.9 & $2.09(2.01-2.18)^{\star \star \star}$ & $1.25(1.20-1.31)^{* \star *}$ \\
\hline \multicolumn{6}{|c|}{ Heart failure } \\
\hline No & 8890 & 260674 & 34.1 & 1.00 & 1.00 \\
\hline Yes & 390 & 6426 & 60.7 & $1.85(1.67-2.04)^{\star \star \star}$ & $1.01(0.91-1.12)$ \\
\hline \multicolumn{6}{|l|}{ CAD } \\
\hline No & 6341 & 215607 & 29.4 & 1.00 & \\
\hline Yes & 2939 & 51493 & 57.1 & $1.98(1.89-2.07)^{\star \star \star}$ & $1.16(1.10-1.22)^{\star \star \star}$ \\
\hline \multicolumn{6}{|c|}{ Medication } \\
\hline \multicolumn{6}{|c|}{ Use of tiotropium } \\
\hline No & 9269 & 266858 & 34.7 & 1.00 & 1.00 \\
\hline Yes & 11 & 241 & 45.6 & 1.30 (0.72 to 2.35 ) & $0.80(0.44-1.44)$ \\
\hline \multicolumn{6}{|c|}{ Use of ipratropium } \\
\hline No & 9240 & 266591 & 34.7 & 1.00 & 1.00 \\
\hline Yes & 40 & 509 & 78.6 & $2.35(1.72-3.20)^{\star \star \star}$ & $1.48(1.08-2.02)^{\star}$ \\
\hline \multicolumn{6}{|c|}{ Annual OPD visits } \\
\hline$<30$ & 5103 & 193353 & 26.4 & 1.00 & 1.00 \\
\hline$\geq 30$ & 4177 & 73746 & 56.6 & $2.21(2.12-2.30)^{\star \star \star}$ & $1.43(1.37-1.50)^{\star \star \star}$ \\
\hline
\end{tabular}

${ }^{*} \mathrm{p}<0.05,{ }^{* \star *} \mathrm{p}<0.001$.

†Multivariate Cox proportional hazards regression model including COPD, age (categorical), diabetes mellitus, dyslipidemia, hypertension, heart failure, CAD, tiotropium use, ipratropium use and annual OPD visits (categorical).

CAD, coronary artery disease; COPD, chronic obstructive pulmonary disease; DM, diabetes mellitus; IR, incidence rate per 1000 personyears; OPD, outpatient department.

The age-stratified results showed that patients with COPD had a significantly higher risk of BPH compared with enrollees without COPD in all age groups. The adjusted HRs of BPH were 1.68 (95\% CI 1.46 to 1.94), 1.51 (95\% CI 1.41 to 1.62 ) and 1.36 (95\% CI 1.27 to 1.45 ) for those aged 40-54, 55-69 and over 70 years, respectively. In the comorbidity-stratified analysis, the association between levels of COPD and BPH was similar, except for heart failure (table 3).

\section{DISCUSSION}

In this large population-based nationwide cohort study, patients with COPD exhibited a 1.53 times higher risk of BPH compared with enrollees without COPD. Although dyslipidemia, hypertension, ipratropium use and annual OPD visits were associated with a higher risk of $\mathrm{BPH}$, the risk of $\mathrm{BPH}$ remained significantly higher in patients with COPD after adjustment for these covariates. According to our review of relevant literature, this is the first cohort 


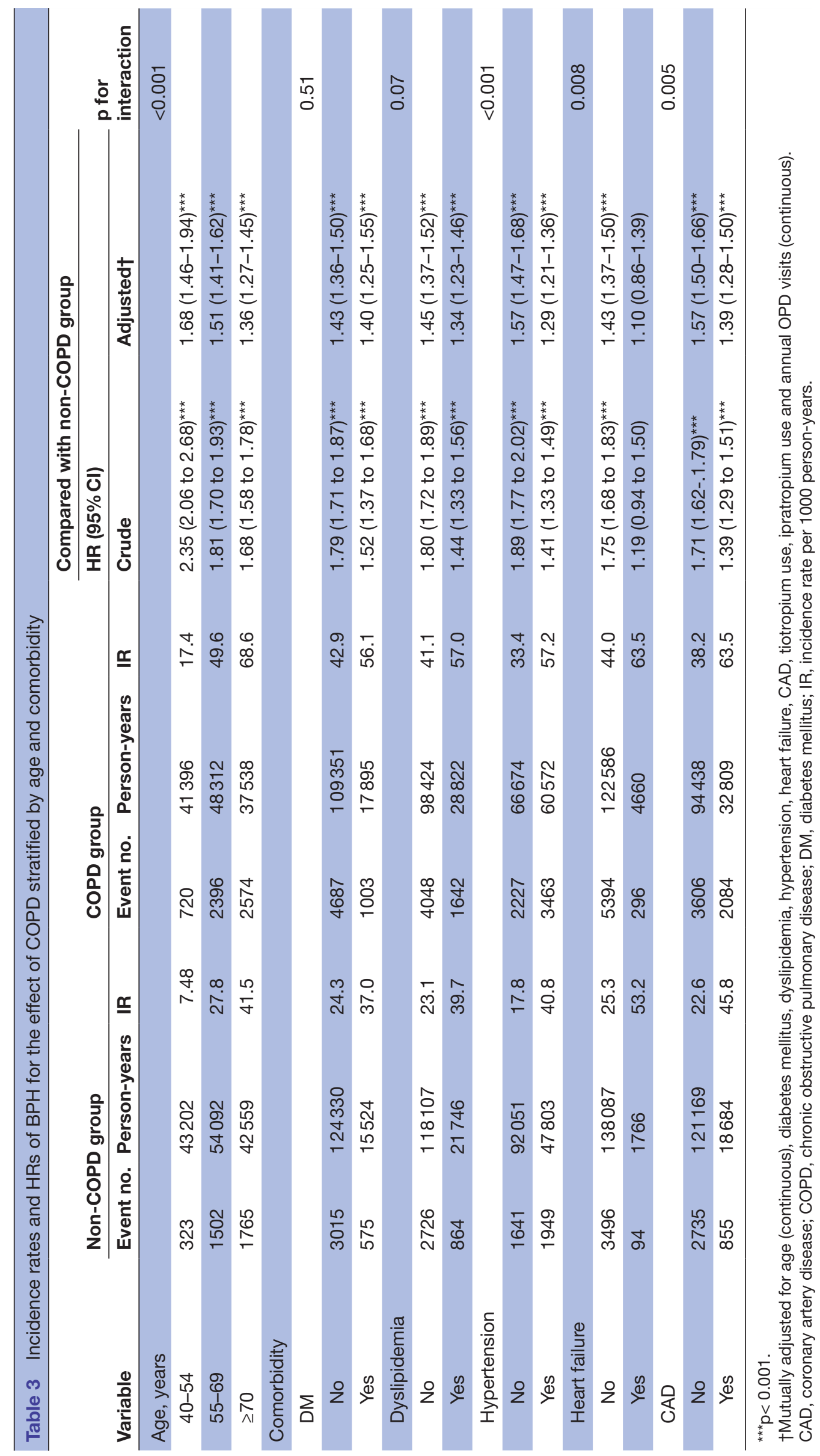


study demonstrating that COPD is associated with increased risk of BPH.

Although the exact underlying mechanisms of the observed relationship between COPD and BPH are unknown, several lines of evidence might offer plausible explanations. First, because inflammation in patients with COPD has been reported in the airway as well as outside the respiratory system, ${ }^{56}$ emerging evidence suggests that inflammation also potentially plays a crucial role in BPH development and progression. ${ }^{13}{ }^{20}$ Higher levels of IL-6 and CRP were found in the serum and induced sputum of patients with COPD. ${ }^{14}{ }^{1521}$ Similarly, Schenk et al reported that high serum levels of CRP and IL-6 were associated with an increased risk of BPH. ${ }^{16}$ Second, Jiang et al reported that activation of the hypoxic-inducible factor (HIF)- $1 \alpha$ pathway by the nuclear factor $\mathrm{KB}$ contributed to the development of COPD in animal models ${ }^{22}$ whereas Kim et al proposed that HIF-1 $\alpha$ mediated BPH under inflammatory conditions in rats, ${ }^{23}$ implying that COPD and BPH might share an underlying pathophysiology. Finally, patients with COPD appeared to be more physically inactive than individuals without COPD and reportedly spent substantially less time walking and standing, compared with sedentary healthy older people. ${ }^{1724}$ A meta-analysis of 11 studies by Parsons et al concluded that moderate to vigorous physical activity was associated with decreased risk of BPH or LUTS. ${ }^{18}$ Thus, we speculate that physical inactivity among patients with COPD is a contributing factor of increased risk of BPH.

In addition to physical activity, other factors associated with increased risk of BPH include diabetes, dyslipidemia, hypertension and obesity. ${ }^{25-27}$ Therefore, we included diabetes, dyslipidemia and hypertension as comorbidities for adjustment. Our analyses were generally consistent with previous studies, ${ }^{25-27}$ suggesting that dyslipidemia and hypertension were associated with increased risk of $\mathrm{BPH}$. To avoid bias in the study results, we did not further adjust for obesity because diabetes, dyslipidemia and hypertension are already highly associated with obesity. ${ }^{28}$ On the other hand, Russo et al found that in a cross-sectional study in a cohort of patients with LUTS/BPH, moderate-to-severe LUTS was independently associated with Framingham cardiovascular risk score of $\geq 10 \%{ }^{29}$ Therefore, we included CAD as a comorbidity for adjustment. Our results appeared to be consistent with the study by Russo et al, suggesting that CAD was significantly associated with increased risk of $\mathrm{BPH}$. The association between cardiovascular diseases and BPH/LUTS merits further investigation.

Patients with $>30$ annual OPD visits had a 1.43 times higher rate of $\mathrm{BPH}$ diagnosis compared with enrollees with fewer than 30 annual OPD visits. To minimise surveillance bias, we used annual OPD visits as a covariate for adjustment. The data revealed that COPD is a predisposing factor independent of annual OPD visits.

Cigarette smoking is a strong predisposing factor for COPD development. By contrast, previous studies have suggested that smokers may be less likely to develop BPH than non-smokers. For example, Kupeli et al reported that the mean prostate volume was lower in smokers than in non-smokers, ${ }^{30}$ and Matzkin et al observed that prostate size did not differ between current smokers, ex-smokers and never-smokers. ${ }^{31}$ Moreover, a recent meta-analysis combining eight observational studies indicated there is no significant association between smoking and $\mathrm{BPH}$ risk either for ex-smokers or current smokers. ${ }^{32}$ Although the NHIRD does not contain information regarding smoking habits of enrollees, a recent study by Cheng et al indicated that approximately $82.9 \%$ of patients with COPD are eversmokers in Taiwan. ${ }^{33}$ A public health report released by the Ministry of Health and Welfare of Taiwan indicated that the ratio of smokers in the male population of Taiwan was $44.3 \%$ in individuals aged $41-45$ years, and $14.9 \%$ in individuals aged over 66 years. ${ }^{34}$ We reasoned that the data from Cheng $e t a l^{33}$ could be attributed to the ratio of smokers in the COPD group; and the data from Ministry of Health and Welfare of Taiwan ${ }^{34}$ could be attributed to the ratio of smoker in the non-COPD group in our study. Altogether, we speculate that although the ratio of smokers is higher in the COPD group than in the non-COPD group, the higher risk of BPH in patients with COPD might not be confounded by mechanisms related to cigarette smoking.

The stratified analysis demonstrated that the incidence of BPH substantially increased with age in both groups. However, the HRs of BPH decreased with an increase in age. This decrease may be because the comorbidities of COPD increased with age, thus attenuating the influence of COPD on $\mathrm{BPH}$ development when we adjusted for comorbidities.

Anticholinergic agents (ipratropium and tiotropium) are extensively used for maintenance treatment in patients with COPD. Previous studies have suggested that anticholinergic agents are associated with acute urinary retention, which implies that these medications might increase the risk of $\mathrm{BPH} .{ }^{35}{ }^{36}$ In our analysis, long-term tiotropium use did not alter the risk of $\mathrm{BPH}$, which was consistent with the study by Miyazaki et al. ${ }^{37}$ By contrast, ipratropium was associated with increased BPH risk. In our study, none of the tiotropium was administered using a nebuliser, whereas some ipratropium was administered using a nebuliser. The association between ipratropium use and BPH risk may be partly explained by ipratropium being administered using a nebuliser, which exerts more systemic effects compared with other forms of anticholinergic agents, such as a dry powder inhaler or a metered dose inhaler. A nested case-control study by Afonso et al suggests that acute urinary retention is associated with the use of inhaled anticholinergic agent, particularly that administered using a nebuliser. ${ }^{36}$ However, in the present observational study, which employed encrypted secondary data, we were unable to access details of each enrollee to examine the adherence of prescribed medications, which may potentially bias our interpretations.

The strength of our study is that we retrieved data from the NHIRD, which is highly representative of the general 
population. Nevertheless, several limitations of our study should be addressed. First, a lack of detailed patient information involving cigarette smoking habits, body mass index, dietary preference and family history of systemic disease in the NHIRD may have yielded biased study results because these may be risk factors or comorbidities of BPH. Second, each diagnosis was based on ICD-9-CM codes obtained from administrative data. Detailed information regarding clinical variables, such as lung function tests, image results, serum prostate-specific antigen and prostate volume were unavailable. Therefore, determining whether more severe COPD further increased the risk of BPH was challenging. Finally, despite our meticulous study design, unknown confounding factors might remain, leading to bias in our study results. However, we believe that the relationship between COPD and BPH demonstrated in the present study is highly reliable because of the database validity, a large sample size and long follow-up periods.

In summary, our study suggests that all patients with COPD aged 40 years and over have a higher future risk of $\mathrm{BPH}$. We suggest that clinicians should be cautious about the increased risk of BPH in patients with COPD. Future research is warranted to validate our results and elucidate the pathophysiology of these two diseases.

\section{Author affiliations}

${ }^{1}$ Department of Public Health, China Medical University, Taichung, Taiwan ${ }^{2}$ Division of Respiratory Therapy, Asia University Hospital, Asia University, Taichung, Taiwan

${ }^{3}$ Department of Respiratory Therapy, China Medical University, Taichung, Taiwan

${ }^{4}$ Division of Chest Medicine, Department of Internal Medicine, Asia University Hospital, Taichung, Taiwan

${ }^{5}$ Department of Biotechnology, College of Medical and Health Science, Asia University, Taichung, Taiwan

${ }^{6}$ Institute of Molecular Biology, College of Life Sciences, National Chung Hsing University, Taichung, Taiwan

${ }^{7}$ Division of Pulmonary and Critical Care Medicine, Department of Internal Medicine, China Medical University Hospital, China Medical University, Taichung, Taiwan

${ }^{8}$ College of Medicine, China Medical University, Taichung, Taiwan

${ }^{9}$ Management Office for Health Data, China Medical University Hospital, Taichung, Taiwan

${ }^{10}$ Department of Respiratory Therapy, China Medical University Hospital, China Medical University, Taichung, Taiwan

${ }^{11}$ Department of Health Care Administration, Asia University, Taichung, Taiwan

Contributors $\mathrm{Y}-\mathrm{HP}, \mathrm{C}-\mathrm{WH}, \mathrm{W}-\mathrm{CL}$ and $\mathrm{W}-\mathrm{CH}$ were involved in conception and design, T-NW and $\mathrm{H}-\mathrm{JC}$ were involved in administrative support, Y-HP, M-CY, Y-MH and $\mathrm{W}-\mathrm{CH}$ were involved in collection and assembly of data. All authors involved in manuscript writing and final approval of the manuscript.

Funding This study is supported in part by Taiwan Ministry of Health and Welfare Clinical Trial and Research Center of Excellence (MOHW106-TDU-B-212-113004), China Medical University Hospital, Academia Sinica Taiwan Biobank Stroke Biosignature Project (BM10501010037), NRPB Stroke Clinical Trial Consortium (MOST 105-2325-B-039-003), Tseng-Lien Lin Foundation, Taichung, Taiwan, Taiwan Brain Disease Foundation, Taipei, Taiwan, and Katsuzo and Kiyo AoshimaMemorial Funds, Japan. The funders had no role in study design, data collection and analysis, decision to publish, or preparation of the manuscript. No additional external funding was received for this study.

Competing interests None declared.

Patient consent Detail has been removed from this case description/these case descriptions to ensure anonymity. The editors and reviewers have seen the detailed information available and are satisfied that the information backs up the case the authors are making.

Provenance and peer review Not commissioned; externally peer reviewed. Data sharing statement № additional data are available.

Open Access This is an Open Access article distributed in accordance with the Creative Commons Attribution Non Commercial (CC BY-NC 4.0) license, which permits others to distribute, remix, adapt, build upon this work non-commercially, and license their derivative works on different terms, provided the original work is properly cited and the use is non-commercial. See: http://creativecommons.org/ licenses/by-nc/4.0/

(c) Article author(s) (or their employer(s) unless otherwise stated in the text of the article) 2017. All rights reserved. No commercial use is permitted unless otherwise expressly granted.

\section{REFERENCES}

1. World Health Organization. The top 10 causes of death. http://www. who.int/mediacentre/factsheets/fs310/en/ (accessed 12 Dec 2016).

2. Negewo NA, McDonald VM, Gibson PG. Comorbidity in chronic obstructive pulmonary disease. Respir Investig 2015;53:249-58.

3. Liao WC, Lin CL, Chang SN, et al. The association between chronic obstructive pulmonary disease and dementia: a population-based retrospective cohort study. Eur J Neurol 2015;22:334-40.

4. Smith MC, Wrobel JP. Epidemiology and clinical impact of major comorbidities in patients with COPD. Int J Chron Obstruct Pulmon Dis 2014;9:871-88.

5. Agusti A, Soriano JB. COPD as a systemic disease. COPD 2008;5:133-8.

6. Fabbri LM, Rabe KF. From COPD to chronic systemic inflammatory syndrome? Lancet 2007;370:797-9.

7. Garraway WM, Lee RJ, Collins GN. High prevalence of benign prostatic hypertrophy in the community. The Lancet 1991;338:469-71.

8. Thorpe A, Neal D. Benign prostatic hyperplasia. Lancet 2003;361:1359-67.

9. De Nunzio C, Aronson W, Freedland SJ, et al. The correlation between metabolic syndrome and prostatic diseases. Eur Urol 2012;61:560-70.

10. Parsons JK, Sarma AV, McVary K, et al. Obesity and benign prostatic hyperplasia:clinical connections, emerging etiological paradigms and future directions.J Urol 2013;189:S102-S106.

11. Sea J, Poon KS, McVary KT. Review of exercise and the risk of benign prostatic hyperplasia. Phys Sportsmed 2009;37:75-83.

12. Shaw JG, Vaughan A, Dent AG, et al. Biomarkers of progression of chronic obstructive pulmonary disease (COPD). J Thorac Dis 2014;6:1532-47.

13. Bostanci Y, Kazzazi A, Momtahen S, et al. Correlation between benign prostatic Hyperplasia and inflammation. Curr Opin Urol 2013;23:5-10.

14. Grubek-Jaworska $\mathrm{H}$, Paplińska M, Hermanowicz-Salamon J, et al. IL-6 and IL-13 in induced sputum of COPD and asthma patients: correlation with respiratory tests. Respiration 2012;84:101-7.

15. Karadag F, Kirdar S, Karul AB, et al. The value of C-reactive protein as a marker of systemic inflammation in stable chronic obstructive pulmonary disease. Eur J Intern Med 2008;19:104-8.

16. Schenk JM, Kristal AR, Neuhouser ML, et al. Biomarkers of systemic inflammation and risk of incident, symptomatic benign prostatic Hyperplasia: results from the prostate Cancer prevention trial. Am J Epidemiol 2010;171:571-82.

17. Spruit MA, Pitta F, McAuley E, et al. Pulmonary Rehabilitation and physical activity in patients with chronic obstructive pulmonary disease. Am J Respir Crit Care Med 2015;192:924-33.

18. Parsons JK, Kashefi C. Physical activity, benign prostatic hyperplasia, and lower urinary tract symptoms. Eur Urol 2008;53:1228-35.

19. National Health Insurance Research Database. Website http://nhird. nhri.org.tw/en/Data Subsets.html (accessed 9 Apr 2017).

20. Donnell RF. Benign prostate Hyperplasia: a review of the year's progress from bench to clinic. Curr Opin Urol 2011;21:22-6.

21. Zhang $\mathrm{Y}$, Bunjhoo $\mathrm{H}$, Xiong W, et al. Association between C-reactive protein concentration and chronic obstructive pulmonary disease: a systematic review and meta-analysis. J Int Med Res 2012;40:1629-35.

22. Jiang $\mathrm{H}, \mathrm{Zhu} \mathrm{Y}, \mathrm{Xu} \mathrm{H}$, et al. Activation of hypoxia-inducible factor1alpha via nuclear factor-kappa $B$ in rats with chronic obstructive pulmonary disease. Acta Biochim Biophys Sin 2010;42:483-8. 
23. Kim HJ, Park JW, Cho YS, et al. Pathogenic role of HIF-1 $\alpha$ in prostate hyperplasia in the presence of chronic inflammation. Biochim Biophys Acta 2013;1832:183-94.

24. Pitta F, Troosters T, Spruit MA, et al. Characteristics of physical activities in daily life in chronic obstructive pulmonary disease. Am J Respir Crit Care Med 2005;171:972-7.

25. Hwang EC, Kim SO, Nam DH, et al. Men with hypertension are more likely to have severe lower urinary tract symptoms and large prostate volume. LUTS: Lower Urinary Tract Symptoms 2015;7:32-6.

26. Chu KF, Rotker K, Ellsworth P. The impact of obesity on benign and malignant urologic conditions. Postgrad Med 2013;125:53-69.

27. Parsons JK, factors $L$. Lifestyle factors, benign prostatic Hyperplasia, and lower urinary tract symptoms. Curr Opin Urol 2011;21:1-4.

28. Kyrou I, Randeva HS, Weickert MO, et al. Clinical Problems Caused by Obesity. In: De Groot LJ, Beck-Peccoz P, Chrousos G, eds. Endotext. South Dartmouth (MA), 2000.

29. Russo Gl, Castelli T, Privitera S, et al. Increase of Framingham cardiovascular disease risk score is associated with severity of lower urinary tract symptoms. BJU Int 2015;116:791-6.

30. Küpeli B, Soygür T, Aydos K, et al. The role of cigarette smoking in prostatic enlargement. Br J Urol 1997;80:201-4.
31. Matzkin H, Cytron S, Simon D. Is there an association between cigarette smoking and gland size in benign prostatic Hyperplasia? Prostate 1996;29:42-5.

32. $\mathrm{Xu} \mathrm{H}, \mathrm{Fu} \mathrm{S}$, Chen Y, et al. Smoking habits and benign prostatic hyperplasia: A systematic review and meta-analysis of observational studies. Medicine 2016;95:e4565.

33. Cheng SL, Chan MC, Wang CC, et al. COPD in Taiwan: a National Epidemiology Survey. Int $J$ Chron Obstruct Pulmon Dis 2015;10:2459-67.

34. Adult Smoking Behavior Surveillance System, Ministry of Health and Welfare of Taiwan, Website: http://tobacco.hpa.gov.tw/Show.aspx? Menuld=581 Acceseed Dec $12,2016,, . .,:$-.

35. Loke YK, Singh S. Risk of acute urinary retention associated with inhaled anticholinergics in patients with chronic obstructive lung disease: systematic review. Ther Adv Drug Saf 2013:4:19-26.

36. Afonso AS, Verhamme KM, Stricker BH, et al. Inhaled anticholinergic drugs and risk of acute urinary retention. BJU Int 2011;107:1265-72.

37. Miyazaki H, Suda T, Otsuka A, et al. Tiotropium does not affect lower urinary tract functions in COPD patients with benign prostatic hyperplasia. Pulm Pharmacol Ther 2008;21:879-83. 